\title{
Acute Gastroenteritis: Contribution of SOS Médecins Network
}

\author{
Marc Ruello*1, Benjamin Larras ${ }^{1}$, Noémie Fortin ${ }^{1}$, Nathalie Jourdan da-silva ${ }^{1}$, Pascal \\ Chansard $^{2}$, Céline Caserio-Schönemann ${ }^{1}$, Vanina Bousquet ${ }^{1}$, Anne Fouillet ${ }^{1}$ and \\ Isabelle Pontais ${ }^{1}$
}

11. French Institute for Public Health Surveillance, Saint-Maurice Cédex, France; ${ }^{2} S O S$ Médecins, Paris, France

\section{Objective}

To illustrate the complementarity and added value of the GP's emergency network "SOS Médecins" through an example of an epidemic of gastroenteritis (GE).

\section{Introduction}

In France, the surveillance of GE is performed by several complementary systems including specific and syndromic surveillance systems.

The GP's emergency associations "SOS Médecins" are part of the French syndromic surveillance system SurSaUD since 2006. SOS Médecins functions as a liberal medical regulation. In 9 years, the network has become almost exhaustive and contribute to the surveillance of seasonal and non-seasonal health events at different geographical scales, in the fields of infectious diseases and environmental health. GE is one of the 50 indicators daily followed by the by the French Institute for Public Health Surveillance (InVS) syndromic surveillance unit.

\section{Methods}

In July 2015, 60 of the 62 associations (about 1000 GP's) transmit daily data to InVS, representing an average daily volume of about 10,000 consultations. National coverage is nearly exhaustive and distribution is homogeneous all over territory including overseas. $80 \%$ of the population living in urban areas is estimated to potentially have access to this type of structure. At a national level, the mean proportion of coding for medical diagnoses is $84 \%$ in 2015 (only $60 \%$ in 2010).

The daily national analysis is performed based on the number of acts, 7 days moving average and the proportion of the tracking indicator among coded acts. Similar analysis are performed at regional level. Data analysis is performed daily and weekly using dashboards and control charts that are generated by a business intelligence application.

\section{Results}

In September 2014, an increase of the proportion of GE diagnoses in all-coded diagnosis was observed at a national level through daily dashbords. The regional analysis revealed that some regions were more impacted such as Normandie and Pays de la Loire (both West-North regions). The early signal was confirmed after querying practionners of SOS Médecins associations in Basse-Normandie. The analysis of the specific surveillance data (GP's in-hour nor laboratory testing) or the syndromic OSCOUR Emergency Department data did not showed any increase in their GE indicators. The syndromic network of Public Health England (PHE) was contacted and reported an increase in the number of cases of GE. The clinical presentation of GE observed in France and UK were mostly upper gastro-intestinal presentation with vomiting symptoms. This episode affected all age groups and particularly children under 15 years old and young adults

\section{Conclusions}

SOS Médecins data was the only data source to identifiy an increase in GE in two French regions.

The clinical presentation did not fit with the various case definitions adopted. In fact, the specific monitoring systems could not detect the increase because they use a restrictive case definition only including acute diarrhea. Also, this outbreak has not been detected in the emergency because symptoms were not serious in all age groups. This episode allowed to discuss the relevance of the different case definitions used in France for GE surveillance. This data source is essential for syndromic surveillance system of InVS: data quality and geographical coverage of the network allows, in addition to emergency services, to obtain accurate analysis epidemic phenomena (eg flu or heat wave)

\section{Keywords}

acute gastro-enteritis; SOS Médecins; GP's

\section{Acknowledgments}

to SOS Médecins associations for providing data

\section{*Marc Ruello}

E-mail: m.ruello@invs.sante.fr 